Renormalization Group for the Octagonal Quasi-Periodic Tiling

This content has been downloaded from IOPscience. Please scroll down to see the full text. 1990 Europhys. Lett. 11439

(http://iopscience.iop.org/0295-5075/11/5/009)

View the table of contents for this issue, or go to the journal homepage for more

Download details:

IP Address: 130.120.231.173

This content was downloaded on 07/04/2015 at $16: 44$

Please note that terms and conditions apply. 
Europhys. Lett., 11 (5), pp. 439-443 (1990)

\title{
Renormalization Group for the Octagonal Quasi-Periodic Tiling.
}

C. Sire $(*)$ and J. BeLlissard $(* *)(* * *)$

(*) Laboratoire de Physique des Solides de Bellevue-CNRS

92195 Meudon Cedex, France

(**) Centre de Physique Théorique, CNRS-Luminy, Case 907-CPT

13288 Marseille Cedex 9, France

(***) Université de Provence - Marseille, France

(received 10 November 1989; accepted 3 January 1990)

PACS. 71.20 - Electronic density of states determinations.

PACS. 71.25 - Nonlocalized single-particle electronic states.

PACS. 71.30 - Metal-insulator transitions.

\begin{abstract}
We study a renormalization group for a tight-binding Hamiltonian on the standard octagonal tiling. Our method can be generalized to any self-similar quasi-crystal in $2 \mathrm{D}$ or even 3D. In the limit of large potentials compared to the hopping parameters, there are numerical evidences that the spectrum is a Cantor set with zero Lebesgue measure.
\end{abstract}

\section{Introduction.}

Since the discovery of quasi-crystals, there has been a growing interest in the study of quasi-periodic tight-binding models. In one dimension by using a transfer matrix formalism, exact renormalization groups (RG) were found [1]. Moreover, an alternative operator treatment can be written [2]. This last procedure is not specific to the $1 \mathrm{D}$ case, whereas other methods, though efficient in $1 \mathrm{D}$, have no generalization in higher dimensions. In $1 \mathrm{D}$, the spectrum measure is zero with an infinite number of gaps [3], whereas in $2 \mathrm{D}$, it was found for a subtiling of the octagonal tiling (with an exact RG) that the spectrum can have any number of gaps and any measure, finite or not [4]. For the Penrose tiling, the spectrum seems to be singular $[5,6]$, and there is no information about the measure. In the following, we apply an approached renormalization procedure to an Hamiltonian $\mathscr{K}$, defined on the standard octagonal quasi-periodic tiling (OQT), which is shown in fig. 1. For recent and general aspects on the OQT see [7] and references therein.

\section{Hamiltonian renormalization.}

The basis of our method is the use of the Schur formula: if $\mathscr{H}^{*}$ is the renormalized Hamiltonian and $\mathscr{D}$ is the projector on the set of vortices of the renormalized OQT, we have

$$
\mathscr{H}^{*}=\mathscr{D} \mathscr{H} \mathscr{D}-\mathscr{D} \mathscr{K} \mathcal{Q} \frac{1}{\mathcal{Q} \mathscr{K} \mathcal{Q}} \mathcal{Q} \mathscr{K} \mathscr{D}, \quad \mathcal{Q}=1-\mathscr{D} .
$$


So, we first define a Hamiltonian $\mathscr{K}$ on the OQT. Then, after setting $\mathscr{H}_{0}=\mathscr{K}-E, E$ is in the energy spectrum if the sequence of Hamiltonians defined by $\mathscr{H}_{n+1}=\mathscr{H}_{n}^{*}$ does not go to a fixed point including infinity. Since the OQT is a self-similar tiling, for $\mathscr{D}$ we choose the projection on the vertices of the standard deflated OQT. Let us stress that this choice is not unique and can turn out not to be the more efficient one. If we start with $\mathscr{H}$ depending on a finite number of parameters (on site potentials, hopping terms), one can see that $\mathscr{H}^{*}$ will depend on an infinite number of such parameters. This is due to the fact that the deflated tiling does not disconnect the OQT (as it occurs in the 1D quasi-periodic chains). However, even if it did, starting with $\mathscr{K}$ depending on a finite number of parameters, the renormalized Hamiltonians would depend on an increasing number of constants at each step. Thus, it seems that this problem cannot be solved exactly. So, in the following section we define a $\mathscr{H}$ for which $\mathscr{K}^{*} \sim \mathscr{K}^{*}$ and $\mathscr{H}^{*}$ has the same kind and number of parameters as $\mathscr{K}$.

\section{Hamiltonian on the OQT.}

Consider the acceptance zone of the OQT, which is the projection of the $4 \mathrm{D}$-hypercube on the orthogonal 2D-space to the physical space (in the cut and project language [8]). This zone can be splitted into seven regions with eight-fold symmetry (fig. 1) which corresponds to five local environments with coordination $3,4,6,7,8$ and two kinds of sites with coordination 5 (fig. 2). For each kind of site $x$ we define an on-site potential $V_{x}$. In addition, we define three kinds of bonds $t, t^{\prime}$ and $r . r$ links the two nearest sites of each rhombus. $t^{\prime}$ links a site $c$ to a $s$ or a $q$, and the $t$ 's are the remaining bonds. Now, applying (1) we have

$$
V_{x}^{*}=V_{y}-t^{2} \sum_{\left\langle y_{1}, y_{2}\right\rangle}\left\langle y_{1}\left|\frac{1}{\mathcal{Q} \mathscr{K} \mathcal{Q}}\right| y_{2}\right\rangle
$$

where $x$ is a site of the deflated OQT, $y$ is the corresponding site in the original OQT (fig. 1)

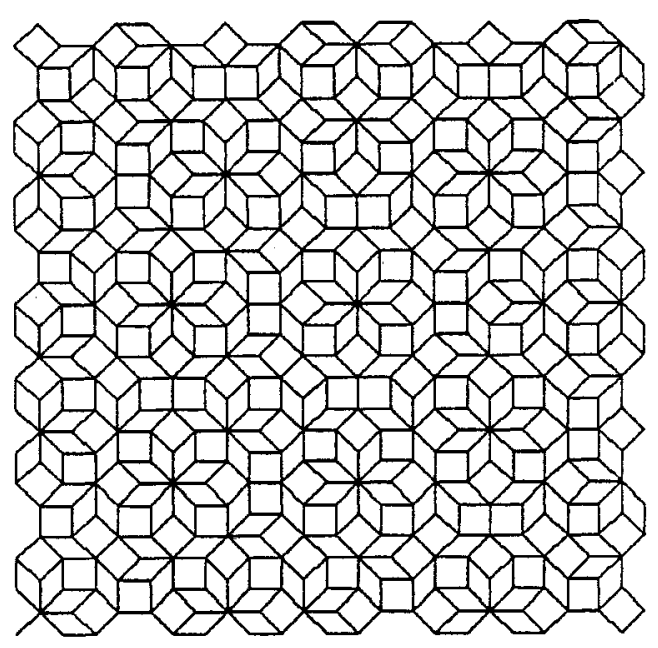

a)

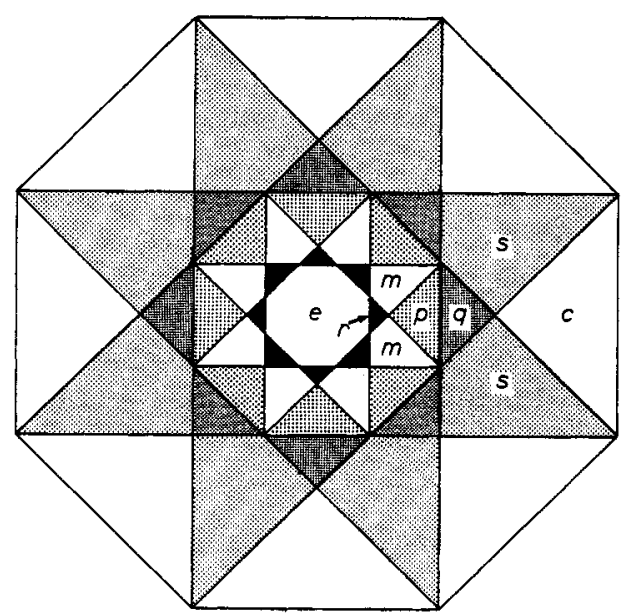

b)

Fig. 1. - a) A finite part of the OQT. b) The octagonal acceptance zone of the OQT. We show the acceptance region for each kind of sites defined in fig. 2. 

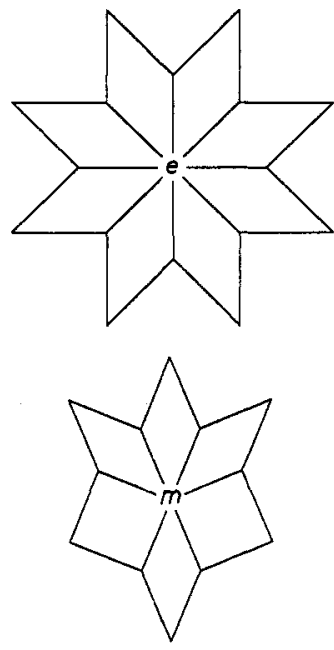
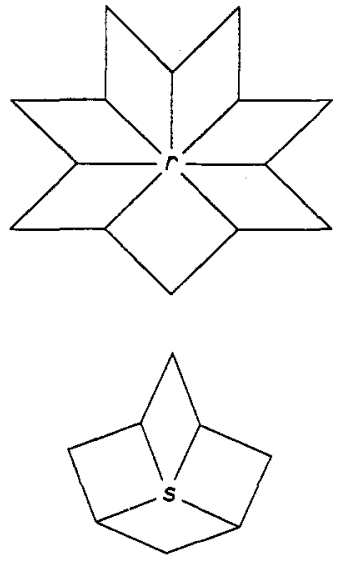
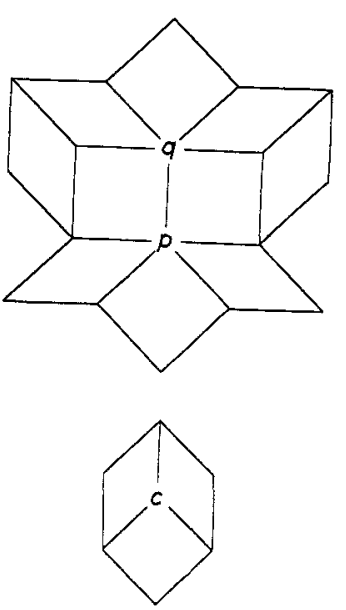

Fig. 2. - The seven kinds of sites $e, r, m, p, q, s, c$.

and $y_{1}$ and $y_{2}$ are the nearest neighbours of $y$, not belonging to the deflated OQT. Our assumption consists in replacing $\mathcal{Q} \mathscr{K} \mathcal{Q}$ by a local Hamiltonian only involving the nearest neighbours of $y$, and the links between them. Thus, we only have to inverse finite-size matrices and then recover exactly the same kind of Hamiltonian after renormalization. We find four relevant Hamiltonians (fig. 3). Finally, we obtain the following results:

$$
\left\{\begin{array}{l}
V_{m}^{*}=V_{p}^{*}=V_{r}^{*}=V_{e}^{*}=V_{e}-\frac{8 t^{2}}{V_{c}+2 r} \\
V_{c}^{*}=V_{p}-t^{2}\left[\frac{1}{V_{q}}+\frac{4}{V_{s}+r}\right] \\
V_{s}^{*}=V_{m}-2 t^{2}\left[\frac{1}{V_{s}+r}+\frac{V_{s}+V_{c}-r}{V_{s}\left(V_{c}+r\right)-r^{2}}\right] \\
V_{q}^{*}=V_{r}-t^{2} \frac{\left(V_{c}^{2}\left(5 V_{s}+2 V_{c}\right)-4 r V_{c}\left(2 V_{s}+V_{c}\right)-r^{2}\left(5 V_{c}+V_{s}-8 r\right)\right)}{V_{c}^{3} V_{s}-r^{2} V_{c}\left(3 V_{s}+V_{c}\right)+2 r^{4}}
\end{array}\right.
$$

The four first renormalized on-site potentials are equal since their corresponding site acceptance zone is included into the acceptance zone of the $e$-sites after renormalization (fig. 1). Moreover, the hopping parameter between two sites of the deflated OQT is derived by means of the same kind of approximation (fig. 3):

$$
\left\{\begin{array}{l}
t^{*}=\frac{2 t^{\prime} t^{2}\left(2 V_{c}-3 r\right)}{V_{s}\left(V_{c}^{2}-r^{2}\right)} \\
t^{*}=\frac{t^{2}}{V_{s} V_{c}^{2}-r^{2}\left(V_{s}+V_{c}\right)}\left[t \frac{\left(V_{c}^{2}-r^{2}\right)}{V_{q}}+t^{\prime}\left(2 V_{c}+\frac{V_{s} V_{c}}{V_{q}}-\frac{r^{2}}{V_{s}}\right)\right] \\
r^{*}=-\frac{2 t^{2}}{V_{s}+r}
\end{array}\right.
$$




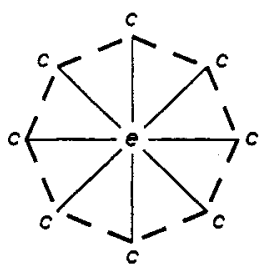

a)

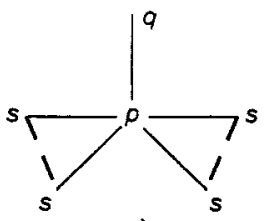

c)

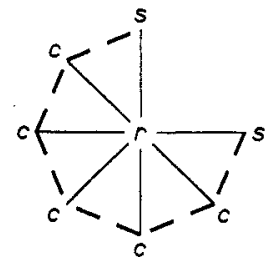

b)

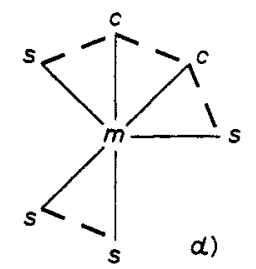

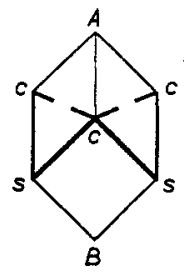

e)

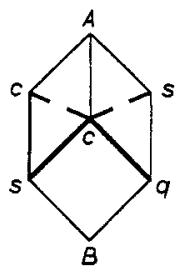

f)

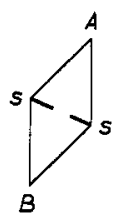

g)

Fig. 3. - The four relevant Hamiltonians are represented by means of the graphs $a$ )- $d$ ). The three kinds of renormalized bonds (linking $A$ and $B$ ) $\left.e) t^{*}, f\right) t^{*}, g$ ) $r^{*}$, and their environment are also shown.

One can show that this $\mathrm{RG}$ is exact if we formally set $t^{2} t^{\prime 2}=t^{4}=t^{\prime 4}=0$. We can even make it simpler by setting $r=0$ in (3), $\left(3^{\prime}\right)$. We have tested our RG on the following model. $V_{x}$ is taken equal to the coordination of site $x$ and we set $t^{\prime}=t, r=0$, and $t$ goes from 0 to -1 (the Laplacian). When we iterate (3), (3') or the projective version of (3), (3') (for instance, we divide all parameters by $V_{e}$ and all renormalized parameters by $\left.V_{e}^{*}\right), E$ is in the spectrum if the iteration of this dynamical system does not go to a fixed point. Numerically, we find that it never occurs so that the spectrum should have zero Lebesgue measure. So, numerically, $E$ is said to be in the $\varepsilon$-spectrum when the iteration with $E-\varepsilon$ and $E+\varepsilon$ goes to two different fixed points, with small $\varepsilon$. In fig. 4 , we show such a spectrum. We see that near $t=0$, the

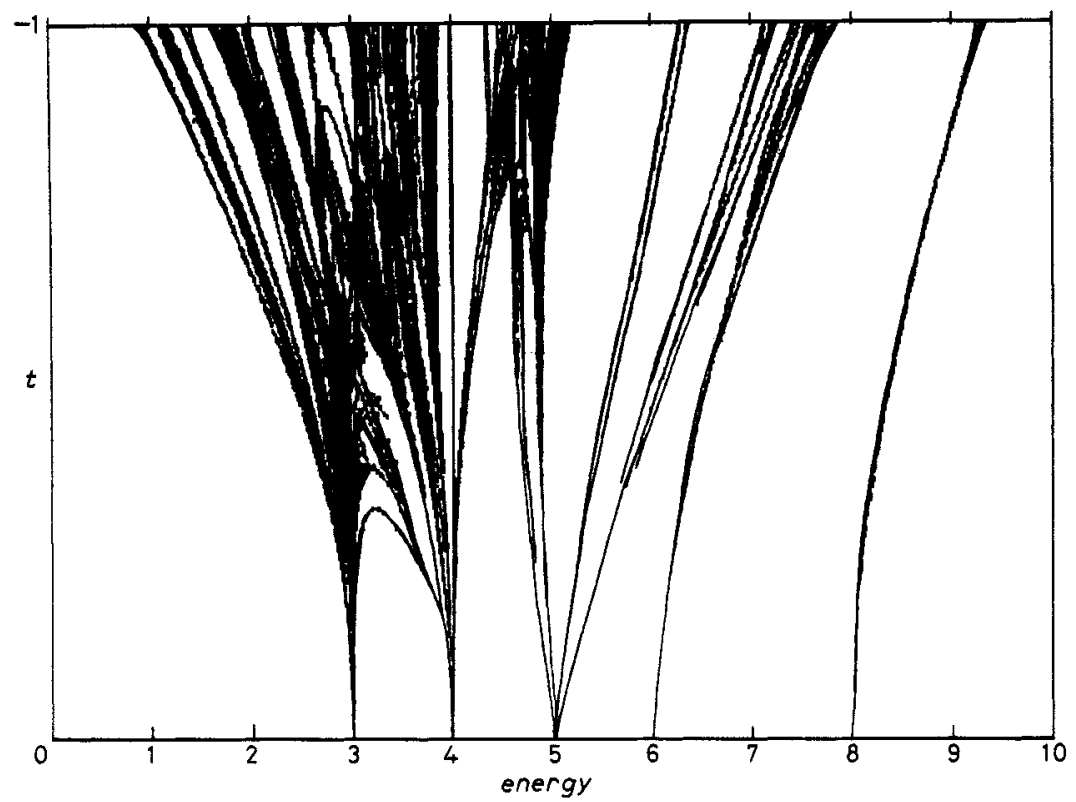

Fig. 4. - The spectrum of the Laplacian-like Hamiltonian defined in sect. 3. 
bands are getting narrower, which is expected since at $t=0$ the OQT splits into an assembly of atoms with energy 3, 4, 5, 6, 7 and 8 . Moreover, the two widest bands correspond to $c$ and $s$ sites which are the most numerous ones, and thus, have the largest effective interaction. Moreover, with our approximation, the band starting from $E=7$ cannot be seen. Since the corresponding environment is the rarest one (the $r$ 's are about 1\%), their effective coupling is very weak. Nevertheless, near $t=1$, even if the sizes of the bands are very consistent with density arguments the lower edge of the spectrum is found to be $E_{\min } \sim 0.8$ instead of the exact value $E_{\min }=0$. (The upper edge is better given.) In order to test numerically our result, it would be necessary to compute the eigenvalues of a large matrix. We insist on the fact that numerical simulations should be made on periodic rational approximant of the OQT [7], in order to minimize boundary effects as those found in [5], for the Penrose tiling (extra molecular state due to undercoordinated sites on the boundary ...).

\section{Conclusion.}

In this paper, we exhibit an approached RG procedure which can be generalized to any self-similar quasi-crystal. In this approximation, we find that the spectrum measure is zero for a Laplacian-like model. One can introduce more renormalization constants and larger effective Hamiltonians to make the results more precise leading to a more complicated dynamical system. The complete study of the fixed points, a more precise RG, and some extra results (for the Penrose tiling) will be addressed in a paper to come.

$$
* * *
$$

It is a pleasure for us to thank M. DuneaU, P. HAUguel and R. Mosseri for fruitful discussions.

\section{REFERENCES}

[1] Konmoto M., Kadanoff L. P. and Tang C., Phys. Rev. Lett., 50 (1983) 1870; Ostlund S., Pandit R., Rand D., Schellnhuber H. J. and Siggia E. D., Phys. Rev. Lett., 50 (1983) 1873.

[2] Bellissard J. and Hauguel P., to be published.

[3] Bellissard J., Iochum B., Scoppola E. and Testard D., to appear in Commun. Math. Phys.

[4] SiRE C., Europhys. Lett., 10 (1989) 483.

[5] Kohmoto M. and Sutherland B., Phys. Rev. B, 34 (1986) 3849.

[6] Odagaki T. and NguYen D., Phys. Rev. B, 33 (1986) 2184; Tsunetsugu H., Fujiwara T., UEDA K. and Tokiniro T., J. Phys. Soc. Jpn., 55 (1986) 1420.

[7] Duneau M., Mosseri R. and Oguey C., to appear in J. Phys. A.

[8] Duneau M. and Katz A., Phys. Rev. Lett., 54 (1985) 2688; Elser V., Acta Crystallogr., Sect. A, 42 (1988) 36; Kalugin P. A., Kitaev A. Y. and Levitov L. S., J. Phys. (Paris) Lett., 46 (1985) L-601. 